Broshkov M. M., Danchuk O. V., Anferova M. V., Dzygal O. F., Yermuraki P. P. Physiological peculiarities of dog' blood erythrocytes in conditions of the Northern Black sea coast. Journal of Education, Health and Sport. 2020;10(2):298-311. eISSN 23918306. DOI http://dx.doi.org/10.12775/JEHS.2020.10.02.035

https://apcz.umk.pl/czasopisma/index.php/JEHS/article/view/JEHS.2020.10.02.035

https://zenodo.org/record/4029222

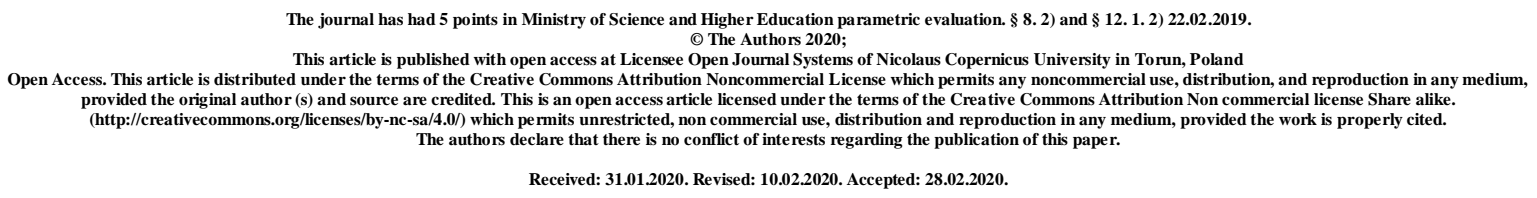

UDC 619:616:41:636.12:611.4

\title{
PHYSIOLOGICAL PECULIARITIES OF DOG' BLOOD ERYTHROCYTES IN CONDITIONS OF THE NORTHERN BLACK SEA COAST
}

\author{
M. M. Broshkovi ${ }^{1}$, O. V. Danchuk ${ }^{1}$, M. V. Anferova ${ }^{1}$, O. F Dzygal $^{2}$, P. P. Yermuraki ${ }^{2}$
}

\author{
${ }^{1}$ Odesa State Agrarian University, Odesa, Ukraine \\ ${ }^{2}$ Odesa National Medical University, Odesa, Ukraine
}

\begin{abstract}
Erythrocyte microscopy is an important step in the study of blood smears, which gives the researcher a lot of information and under certain conditions allows one to make a reliable diagnosis. This article presents new scientific data on the physiological characteristics of erythrocytes in dogs, the variability of their size and shape depending on age, sex and season of the year. The experiment was performed on 147 clinically healthy dogs of different ages, breeds and genders. Blood smears were stained by the Romanowski-Gimse method and subjected to light microscopy with oil immersion. It was found that physiological anisocytosis occurs in $34 \%$ of dogs, and $86 \%$ are unknown poikilocytosis, $10 \%$ - moderate and only $4 \%$ severe. If we analyze the changes in cell size, macrocytic anisocytosis was observed more often in the blood smear of dogs (in 19\% of cases), slightly less - macrocytic (14\%) and only in one case megalocytic anisocytosis was detected. Every third case of anisocytosis was reported in dogs under 6 months of age. In spring, changes in the size of erythrocytes were detected in $59 \%$ of cases (in 10 dogs out of 17), in summer and autumn - 17-18\% and in winter in $8 \%$ of the studied animals. Only $60 \%$ of the studied dogs were found to have normochromia by laboratory analysis. Moreover, hypochromia is 4.7 times more common
\end{abstract}


than hyperchromia. Hyperchromia in dogs was more often recorded in spring (18\%), hypochromia in autumn and spring (41-45\%), and polychromia in summer (24\% of the total number of studied dogs). Inclusions in the erythrocytes of dogs were found in $32 \%$ of experimental blood smears, with $85 \%$ of cases being Jolie bodies, $7.5 \%$ - Kebot rings, $5.6 \%-$ Heinz bodies and $1.9 \%$ - pseudo-inclusions. Physiological poikilocytosis was detected in $47.6 \%$ of blood smears of dogs, out of which in $35.4 \%$ of samples there was a slight poikilocytosis, in $11.6 \%$ - moderate and up to $1 \%$ - severe. The ones that were more frequently encountered were target cells and stomatocytes (14\% each), acanthocytes (11\%), echinocytes (5\%), dacrinocytes (4\%), spherocytes (3\%), keratocytes (2\%), schistocytes (2\%), crescent-shaped cells (1\% of the total number of studied smears). Poikilocytosis in dogs is more common in spring (82\% of smears), slightly less in summer (up to 35\%) and autumn $(21 \%)$.

Key words: dogs; erythrocytes; microscopy; anisocytosis; poikilocytosis.

Introduction. Assessment of erythrocytes is an important step in the study of blood smears. In a systematic approach, it provides the researcher with a lot of information and, under certain conditions, allows a specific diagnosis to be made without further research [1]. Erythrocyte microscopy allows to characterize different species of animals taking into account the diameter, circumference and surface of erythrocytes. Clinicians often have to deal with various hematological syndromes, the most common of which is anemia. From the morphometric parameters of erythrocytes, blood droplets can characterize animal species [2].

Erythroid lineage cells vary widely within the animal kingdom in size, number, shape, lifespan, metabolism, and reactivity [3]. Dog erythrocytes are relatively large and, compared to other animal species, have more light in the center (in the smear), indicating a greater depth in the center of the cell compared to the cells of other animals [4].

The wide range of different physiological forms of normal erythrocytes in different species is a challenge for the researcher. A complete understanding of the physiological characteristics of erythroid cells in the blood of both animals and humans creates the preconditions for the correct identification and interpretation of abnormalities [5]. Normally, a small number of polychromatophilic erythrocytes ( $<1.5 \%$ reticulocytes) and single nuclear erythrocytes are observed in the blood of dogs. The lifespan of erythrocytes in dogs ranges from 110 to 120 days which is 1.5 times longer than in cats [2].

Various causes can affect the shape, size of erythrocyte staining, including regenerative response, metabolic changes, oxidative stress, immune responses, mechanical 
fragmentation, sepsis, toxins, and drugs [6]. Not all of these reasons are pathology. Thus, the physiological causes that can affect erythrocytes include physiological condition (pregnancy, stress), gender, age, living conditions (including oxygen pressure in the environment, temperature), etc. [7]. There are lots of possible intermediate forms of erythrocytes with pronounced morphological features that can be observed in various physiological or pathological conditions [8].

Despite the rapid development of technology in clinical laboratory studies, with advances in flow cytometry and molecular analysis, morphological analysis of a patient's blood smear remains the basis for hematological diagnosis $[9,10]$. Numerous studies indicate that blood smear analysis often provides key information for direct and differential diagnosis in both human and veterinary medicine [11-13]. Scientists and practitioners assessing red blood cells by size, shape, color, distribution or presence of inclusion bodies receive information not only about primary and secondary hemopathy, but also about the state of the whole organism $[14,15]$. There are models for diagnosing anemia that allow it to be detected in a few weeks of clinical manifestation [16]. However, the mechanisms that control the quantitative and qualitative characteristics of erythrocytes and their changes in different conditions are insufficiently studied.

Upon release from the bone marrow, RBCs experience a decrease in both volume and total hemoglobin by an unknown mechanism [17, 18]; after 20-120 days, reacting to an unknown trigger, they are removed. We used the theory of statistical physics and the data of the hospital clinical laboratory [19] to develop a basic equation model for RBC maturation and clearance. The model accurately identifies patients with anemia and distinguishes thalassemic anemia from iron deficiency anemia. More generally, we demonstrate how clinical laboratory data can be used to develop and test a dynamic model of human pathophysiology with potential clinical utility.

Thus, the study of the morphology of erythrocytes of dogs will provide new scientific data on the physiology of erythrocytes in this species, the physiological variability of their size and shape depending on age, sex and season. This will facilitate interpretation and reduce the percentage of erroneous conclusions under smear microscopy and will be useful to both scientists and veterinarians. Therefore, this article is devoted to the morphological description of erythrocytes in dogs.

The aim of the study - to investigate the physiological features of erythrocyte morphology in dogs of different ages, sexes and at different times of the year in the northern Black Sea region. 


\section{Materials and methods}

The study was performed on clinically healthy dogs of different ages, breeds and genders. A total of $147 \mathrm{dog}$ blood smears were analyzed. Blood smears were subjected to light microscopy with oil immersion. Blood smears were stained by the Romanowski-Gimse method [20]. Under conditions of high-quality staining of blood smears, erythrocytes were pink, the cytoplasm of leukocytes and lymphocytes - blue, and their nuclei - dark purple (Fig. 1). Blood products obtained from dogs were the subject of cytological analysis to determine the qualitative characteristics of red blood cells (size, shape, inclusion, etc.). The material was obtained on the basis of private veterinary clinics in Odessa during routine clinical examinations.

Experimental studies were provided according to the Law of Ukraine № 3447-IV of 21.02.06 "On the protection of animals from cruel treatment" and are consistent with the basic principles of the "European Convention for the protection of vertebrate animals used for experimental and scientific purposes" (Strasbourg, 1986), the Declaration on the Humane Treatment of Animals (Helsinki, 2000) and the National Congress on Bioethics "General Ethical Principles of Animal Experimentation" (Kyiv, 2001).

\section{Results}

It is known that dog erythrocytes are larger in size than other species, their diameter ranges from 6 to $8 \mu \mathrm{m}$ [21]. Physiology of morphological changes of erythrocytes is determined by age, gender and breed. Our studies found that the number of erythrocytes in the blood of dogs depending on age, physiological condition, gender and season ranged from 3.7 to $6.9 \mathrm{~T} / 1$, and their diameter ranges from 5 to 8 microns, which is consistent with the norm obtained by other researchers [22].

Morphometric characteristics of erythrocytes are largely determined by age and breed. The change in the size of erythrocytes in a blood smear (anisocytosis) may be associated with an increase in the size of erythrocytes, a decrease in their size, or the appearance in the smear of a combination of both these forms [23]. Fetal erythrocytes are larger than those in adult animals, their diameter decreases during the first 9-12 weeks of life due to replacement by postnatal erythrocytes [24]. Regarding breed characteristics, poodles have constitutional macrocytosis, and some Japanese breeds (Akita, Shiba) have natural (physiological) microcytosis [21]. 


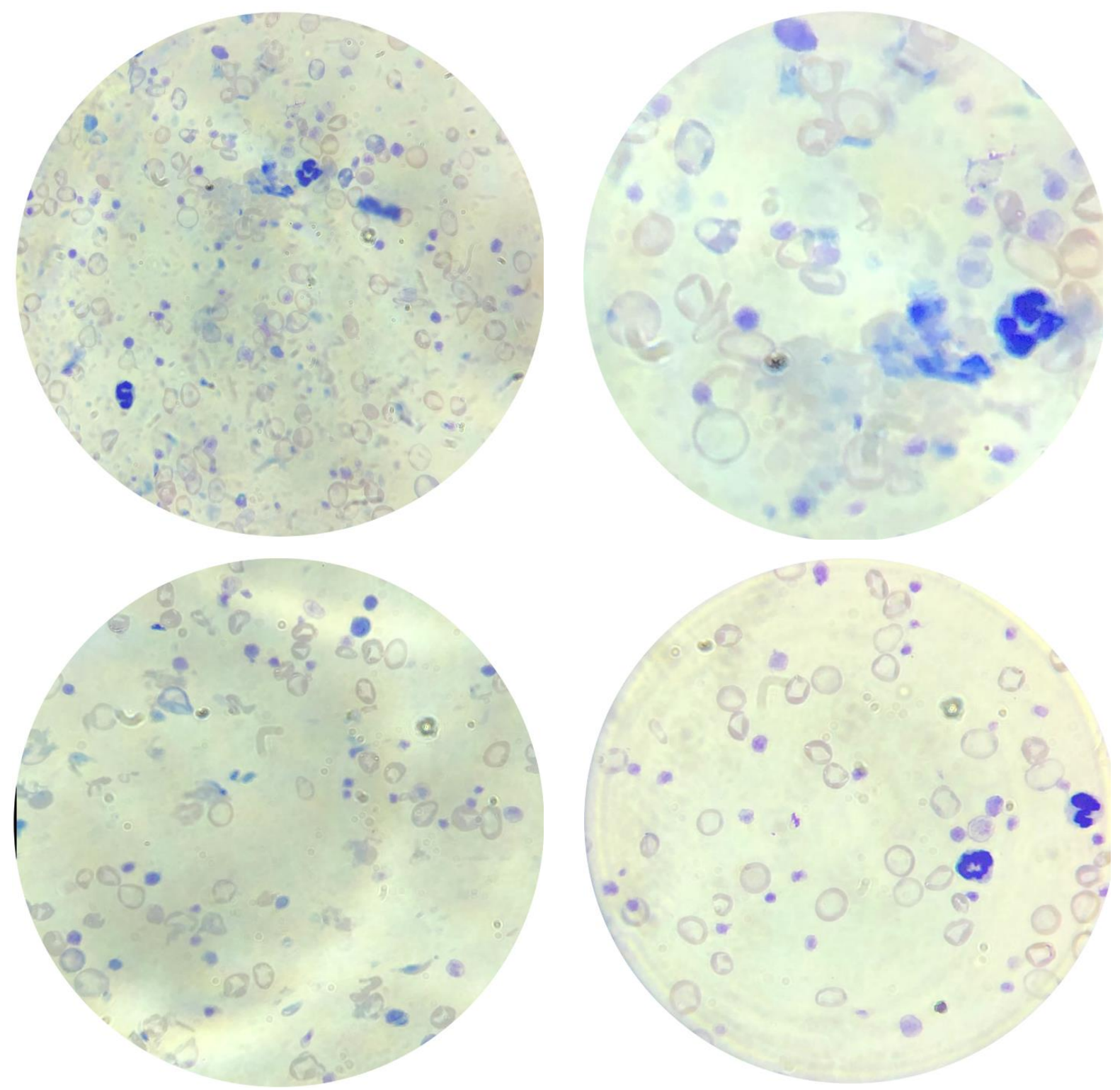

Fig. 1. Blood smears of dogs with different forms of erythrocytes.

Physiological anisocytosis is defined as a change in the size of erythrocytes of dogs without dysfunction of the oxygen transport system, depending on the breed and age, it is diagnosed in $28-89 \%$ of dogs [25]. Of the 147 blood samples from dogs, we found signs of physiological anisocytosis in 34\% of smears, of which: 86 - unknown; $10 \%$ - moderately pronounced; $4 \%$ - severe anisocytosis. If we analyze the changes in cell size, macrocytic (in $19 \%$ of cases) was observed more often, slightly less - microcytic (14\%) and only in one case megalocytic anisocytosis was detected (Table). 
Table

Morphological features of erythrocytes in the blood of dogs

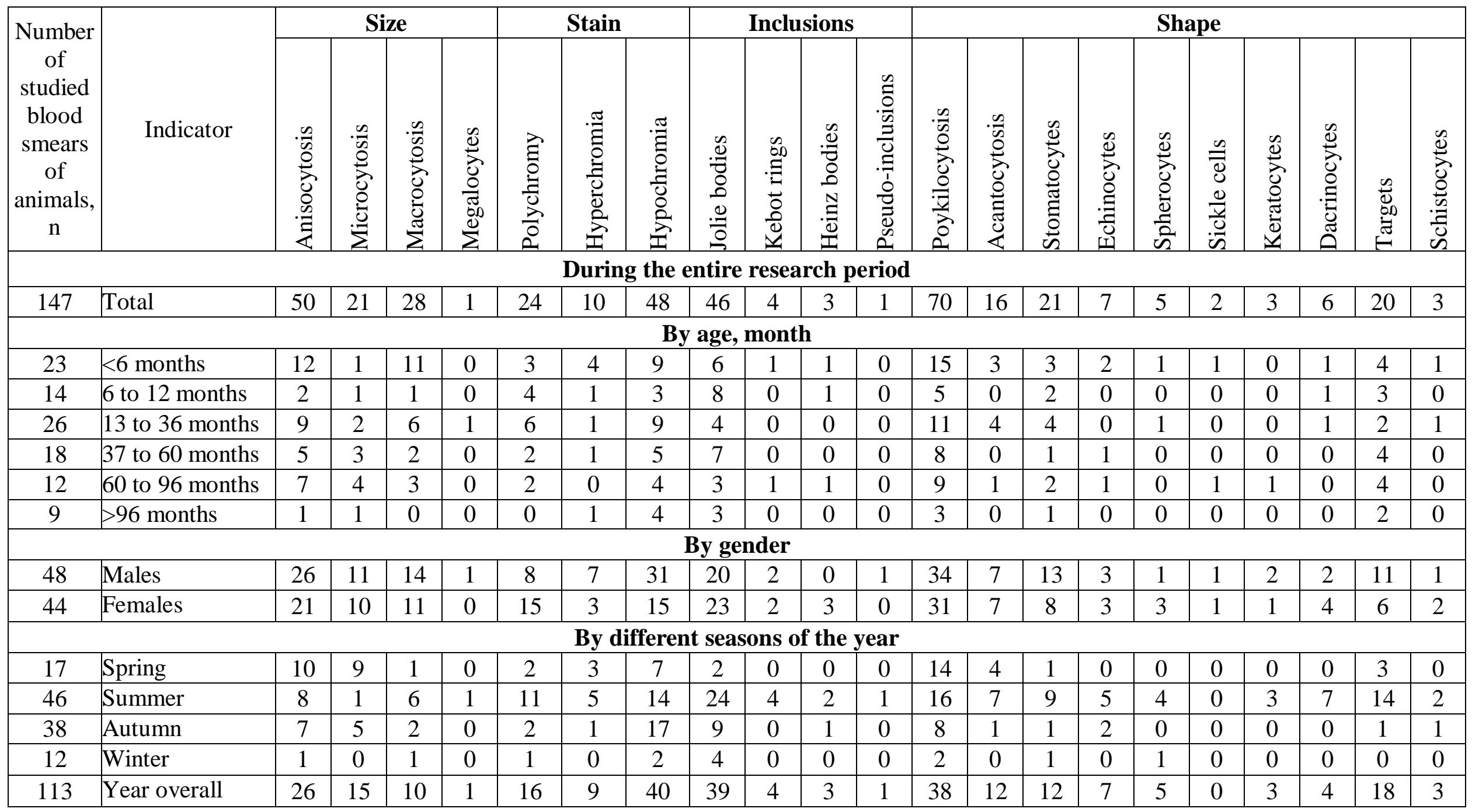


The gender of dogs does not significantly affect the size of erythrocytes in their blood, although physiological anisocytosis in females, compared with males is $13.5 \%$ more common. Moreover, if in females micro- and macrocytosis are detected in the same number of cases (7\% each), then in males macrocytic (10\%) and less microcytic physiological anisocytosis $(6 \%)$ are more often noted.

The age of the animals significantly affects the manifestation of anisocytosis in dogs, so, every third case of anisocytosis was observed in dogs under 6 months of age. It was previously established that changes in the qualitative and quantitative characteristics of red blood of two-month-old puppies affect antibody production during the introduction of a biological stimulus [26]. It should be noted that if young animals are more likely to have macrocytic anisocytosis ( 9 out of 10 cases), then older animals are more likely to have the microcytic one (5-6 out of 10 cases).

For the first time, a significant effect of the season on the frequency of detection of physiological anisocytosis in dogs was established. Thus, in spring changes in the size of erythrocytes were found in 59\% (in 10 dogs out of 17), in summer and autumn - 17-18\% and in winter in $8 \%$ of the studied animals. Microcytic anisocytosis is more common in spring and autumn, and macrocytic anisocytosis in summer and winter. Microcytic anisocytosis is a sign of iron deficiency in the body, which is more often found along with vitamin deficiency in the spring [27].

Thus, in the spring, $90 \%$ of cases of anisocytosis in dog smears were microcytic type. There are data on the higher incidence of iron deficiency anemia in humans in spring and summer than in winter, which researchers associate with seasonal fluctuations in folate and vitamin $\mathrm{B}_{6}[28]$.

However, in domestic dogs, the diet depends little on the season, so the reasons for the seasonal change in the morphology of erythrocytes today remain unknown.

According to the literature, it is known that the concentration of hemoglobin in normochromic erythrocytes is $32-36 \%$ [23]. However, our research found that only $60 \%$ of the dogs tested had laboratory tests for normochromia, and hypochromia was five times more common than hyperchromia.

The presence of hypochromic mature erythrocytes and reticulocytes in the blood smear is a sign of a decrease in hemoglobin in these cells and a direct consequence of the imbalance between the need and the presence of iron in the body [29]. If hypochromia is more associated with iron or vitamin deficiency, hyperchromia greater than $10 \%$ is associated with both hereditary and acquired erythrocyte membrane disorders [30]. Among all reported cases, 
hyperchromia was more commonly diagnosed in dogs under 6 months of age (39\%) and older than 96 months (44\%), and less in dogs aged 6-12 months (21\%) and 37-60 months (28\%). . The dynamics was similar with the frequency of hypochromia. Hyperchromia in dogs was more often recorded in spring (18\%), hypochromia in autumn and spring (41-45\%), and polychromia in summer ( $24 \%$ of the total number of studied dogs). It is difficult to explain the seasonality of erythrocyte chromium changes, but these data are consistent with the available information on the seasonal dynamics of transfer and iron fluctuations in human blood [31]. Regarding the degree of manifestation of erythrocyte chromia, it can be stated that among all animals with signs of hypochromia: minor hypochromia was detected in $73 \%$ of cases; moderate - in $21 \%$; and is expressed in only $6 \%$ of dogs. In addition, the hyperchromia detected in 80 cases was insignificant and in the remaining $20 \%$ moderate.

Inclusions in erythrocytes can occur for various reasons. Their correct identification makes it possible to identify the physiological and pathological state of the dog's body that affects erythropoiesis [3]. In stained smears, erythrocytes are unstructured and do not contain inclusions, however, under certain conditions they show basophilic granularity (under physiological conditions, this occurs infrequently - up to 4 per 10,000 cells). In dog erythrocytes, inclusions were found in almost every third blood smear, with $85 \%$ of cases being Jolie bodies, 7.5\% Kebot rings, 5.6\% Heinz bodies, and 1.9\% pseudoinclusions. Jolie bodies are remnants of the nucleus preserved in erythrocytes as a result of disruption of the process of depletion of oxyphilic normoblast (Harvey, 2011). It should be noted that $98 \%$ of cases of visualization of Jolie's bodies had the character of insignificant, and only $2 \%$ - the expressed detection.

Regarding the age features of detection of inclusions in erythrocytes of dogs, the greater presence (twice) of them in dogs aged 6 to 12 months compared to other age groups is noteworthy, while in dogs aged 13-36 months they were only detected in 15\% of animals. Moreover, in bitches of the body of Jolie in erythrocytes are found $23.8 \%$ more often than in males. In addition, the seasonality of the frequency of diagnosis of inclusions in erythrocytes of dogs was revealed, in particular, in summer and winter they were detected 2-4 times more than in spring and autumn.

The term "poikilocyte" is a general term to describe erythrocytes with an abnormal shape. Poikilocytes can be classified according to specific changes in shape, some of which have a very unique diagnostic value, and other forms are quite nonspecific [32]. Physiological poikilocytosis is characterized by the presence of altered erythrocytes in the peripheral blood. The change in the shape of erythrocytes was detected in $47.6 \%$ of blood smears of dogs, of 
which in $35.4 \%$ of samples there was a slight poikilocytosis, in $11.6 \%$ - moderate and up to $1 \%$ - severe. Target cells and stomatocytes (14\% each), acanthocytes (11\%), echinocytes (5\%), dacrinocytes (4\%), spherocytes (3\%), keratocytes (2\%) crescent-shaped cells (1\% of the total) number of studied smears). There are data that schistocytes are observed in $60-75 \%$ of dogs, but they are rarely found in other species [23] but we found these cells in only $2 \%$ of the studied smears.

There are data on pathophysiological mechanisms that affect the prevalence and type of natural poikilocytosis. In the blood of $31 \%$ of rabbits there are acanthocytes and in $27 \%-$ echinocytes (fragmentation and acanthocytosis were more pronounced in rabbits with inflammatory disease), in particular echinocytosis was associated with renal failure, and serum cholesterol correlated with the percentage of acanthocytes [33]. In contrast, we found no significant association between the onset of poikilocytosis and the incidence of various pathological conditions in dogs.

Physiological poikilocytosis is mainly found in dogs in spring (82\% of smears) and summer (up to $35 \%$ ), while in spring it was detected in only $21 \%$ of smears, and in winter in 12 samples of poikilocytes were not detected. Sexual features of the manifestation of poikilocytosis in dogs have not been established, only a slightly higher percentage of detections of stomatocytes and target cells in males compared to females.

Researchers report [34] that the main causes of anemia in dogs, with the exception of acute hemorrhage, are: chronic dyshemopoietic anemia (PCV <37\%; $\mathrm{n}=456$ ); cancer-related anemias (33.1\%); anemia with inflammatory processes (28.5\%). A study of 147 blood smears of dogs of different ages, sexes and breeds revealed signs of anemia in $13 \%$ of animals, and it was recorded mainly in animals under 6 months of age (almost half of the cases). It is obvious that after birth, fetal erythroid cells of the fetus are replaced by postnatal erythrocytes [35]. Postnatal oxidative stress promotes the rapid replacement of the pool of red blood cells by intensifying their aging, the mechanism of which is free-radical in nature [36].

Due to the deficiency of plastic resources (iron, essential amino acids), biologically active substances (cobalt, B vitamins, etc.), the prolongation of stress in the body of puppies at an early age, the removal of fetal blood cells from the bloodstream is not fully compensated by the arrival of young erythrocytes resulting in an anemic condition [37]. Blood smears of such puppies were characterized by hypochromia of erythrocytes, the presence of shadows of erythrocytes, poikilocytosis, macrocytosis, the presence of inclusions in erythrocytes (Jolie bodies). 
Regarding the gender features of the manifestation of anemia in dogs, at a young age (up to six months of age) it was more often found in males than in bitches, and vice versa - in animals older than one year. This can be partly explained by the higher growth rate of males at a young age [38], as a result of which hematopoiesis (especially with a deficiency of iron and other limiting components and increasing body oxygen demand) does not provide sufficient red blood cells in the bloodstream. There is evidence that people under 11-14 years of age do not have gender features of anemia, but older people are more likely to have anemia, especially women $[39,40]$, which is due to their physiological features.

Our results confirm the need for careful analysis of erythrocyte morphology in research and clinical diagnosis.

\section{Conclusions}

1. Physiological anisocytosis occurs in $34 \%$ of dogs. Among which $86 \%$ are unknown, $10 \%$ - moderate and $4 \%$ - severe poikilocytosis. Microcytic anisocytosis is diagnosed more often (19\%) and macrocytic (14\%) less often. Every third case of anisocytosis was reported in dogs under 6 months of age. In the spring of the year, changes in the size of erythrocytes were found in $59 \%$ of dogs, in summer and autumn $-17-18 \%$ and in winter $-8 \%$ of the studied animals.

2. Normochromia of erythrocytes was detected in $60 \%$ of the examined dogs by laboratory analysis. Hypochromia is 4.7 times more common than hyperchromia. Hypochromia is more common in autumn and spring (41-45\%), hyperchromia in spring $(18 \%)$, and polychromia in summer $(24 \%)$.

3. Inclusions in erythrocytes were found in $32 \%$ of the studied blood smears of dogs, of which $85 \%$ - Jolie bodies, $7.5 \%$ - Kebot rings, 5.6\% - Heinz bodies and $1.9 \%$ - pseudoinclusions.

4. Physiological poikilocytosis was diagnosed in $47.6 \%$ of blood smears of dogs. Among these samples, $35.4 \%$ - minor, $11.6 \%$ - moderate and 1\% - severe poikilocytosis. The ones that were more frequently encountered were target cells and stomatocytes (14\% each), acanthocytes (11\%), echinocytes (5\%), dacrinocytes (4\%), spherocytes (3\%), keratocytes $(2 \%)$, schistocytes $(2 \%)$, crescent-shaped cells $(1 \%)$. Poikilocytosis in dogs is more common in the spring (82\% of smears), less in the summer (up to 35\%) and autumn $(21 \%)$. 


\section{References}

1. Allison R.W., Meinkoth J.H. Hematology without the numbers: in-clinic blood film evaluation. Veterinary Clinics of North America: Small Animal Practice. 2007; 7(2): $245-266$.

2. Adili N., Melizi M., Belabbas H. Species determination using the red blood cells morphometry in domestic animals. Veterinary World. 2016; 9(9): 960-963.

3. Barger A.M. Erythrocyte morphology. Schalm's Veterinary Hematology. $2010 ; 144-151$.

4. Harvey J.W., Harvey J.W. Evaluation of Erythrocytes. Veterinary Hematology. 2012; 49-121. https://doi.org/10.1016/B978-1-4377-0173-9.00004-X

5. Christopher M.M. Erythrocyte shape abnormalities: visual clues to systemic biochemical changes. Proc. of the $11^{\text {th }}$ Eur Soc of Veterinary Clinical Pathology Conf. 2009; 33-40.

6. Pierre R.V. Red cell morphology and the peripheral blood film. Clinics in Laboratory Medicine. 2002; 22(1): 25-61.

7. Anferova M.V., Broshkov M.M., Danchuk O.V. The ratio of erythrocyte populations in the blood of puppies during postnatal oxidative stress. Scientific Bulletin of Veterinary Medicine. - 2019. - N 2. - P. 81-87 [In Ukrainian].

8. Gavazza A., Ricci M., Brettoni M., Gugliucci B., Pasquini A., Rispoli D., Bernabò N., Lubas G. Retrospective and Prospective Investigations about "Quatrefoil" Erythrocytes in Canine Blood Smears. Veterinary Medicine International. 014, 409573. https://doi.org/10.1155/2014/409573

9. Barns S. Numerical modelling of red blood cell morphology and deformability. Queensland University of Technology. 2018: 293 p.

10. Ford J. Red blood cell morphology. International Journal of Laboratory Hematology. 2013; 35(3): 351-357.

11. Diez-Silva M., Dao M., Han J., Lim C.-T., Suresh S. Shape and biomechanical characteristics of human red blood cells in health and disease. MRS Bulletin. 2010; 35(5): $382-388$.

12. Liebman H.A., Weitz I.C. Autoimmune hemolytic anemia. The Medical Clinics of North America. 2017; 101(2): 351-359.

13. Lin Y.-H., Huang S.-S., Wu S.-J., Sung K.-B. Morphometric analysis of erythrocytes from patients with thalassemia using tomographic diffractive microscopy. Journal of Biomedical Optics. 2017; 22(11): 116009. 
14. Ciesla B. Hematology in practice. FA Davis, 2018: 320 p.

15. Reagan W.J., Rovira A.R.I., DeNicola D.B. Veterinary hematology: atlas of common domestic and non-domestic species. John Wiley \& Sons, 2019: 405 p.

16. Higgins J.M., Mahadevan L. Physiological and pathological population dynamics of circulating human red blood cells. Proc. of the National Academy of Sciences of the United States of America. 2010; 107(47): 20587-20592. https://doi.org/10.1073/pnas.1012747107

17. Lew V.L., Raftos J.E., Sorette M., Bookchin R.M., Mohandas N. Generation of normal human red cell volume, hemoglobin content, and membrane area distributions by "birth" or regulation? Blood. 1995; 86(1): 334-341.

18. Waugh R.E., Narla M., Jackson C.W., Mueller T.J., Suzuki T., Dale G.L. Rheologic properties of senescent erythrocytes: loss of surface area and volume with red blood cell age. Blood. 1992; 79(5): 1351-1358.

19. d'OnofrioG., Chirillo R., Zini G., Caenaro G., Tommasi M., Micciulli G. Simultaneous measurement of reticulocyte and red blood cell indices in healthy subjects and patients with microcytic and macrocytic anemia. Blood. 1995; 85(3): 818-823.

20. Miller J.M., Binnicker M.J., Campbell S., Carroll K.C., Chapin K.C., Gilligan P.H., Gonzalez M.D., Jerris R.C., Kehl S.C., Patel R. A guide to utilization of the microbiology laboratory for diagnosis of infectious diseases: 2018 update by the Infectious Diseases Society of America and the American Society for Microbiology. Clinical Infectious Diseases. 2018; 67(6): e1-e94.

21. Meinkoth J.H., Clinkenbeard K.D., Rizzi T.E. Schalm's Veterinary Hematology. 2000: 320 p.

22. Khan S.A., Epstein J.H., Olival K.J., Hassan M.M., Hossain M.B., Rahman K., Elahi M.F., Mamun M.A., Haider N., Yasin G. Hematology and serum chemistry reference values of stray dogs in Bangladesh. Open Veterinary Journal. 2011; 1(1): 13-20.

23. Harvey J.W. Veterinary Hematology-E-Book: A Diagnostic Guide and Color Atlas. Elsevier Health Sciences. 2010: 430 p.

24. Brun-Hansen H.C., Kampen A.H., Lund A. Hematologic values in calves during the first 6 months of life. Veterinary Clinical Pathology. 2006; 35(2): 182-187.

25. Aniołek O., Barc A., Jarosińska A., Gajewski Z. Evaluation of frequency and intensity of asymptomatic anisocytosis in the Japanese dog breeds Shiba, Akita, and Hokkaido. Acta Veterinaria Brno. 2018; 86(4): 385-391. 
26. Broshkov M.M. Production of specific antibodies in the puppy depending on different hematological parameters. Agrarian Bulletin of the Black Sea Coast. Veterinary Sciences. 2014; 72: 12-16 [In Ukrainian].

27. Ronnenberg A.G., Goldman M.B., Aitken I.W., $\mathrm{Xu} \mathrm{X}$. Anemia and Deficiencies of Folate and Vitamin B-6 Are Common and Vary with Season in Chinese Women of Childbearing Age. The Journal of Nutrition. 2000; 130(11): 2703-2710. https://doi.org/10.1093/jn/130.11.2703

28. Li, Q., Liang, F., Liang, W., Shi, W., \& Han, Y. (2019). Prevalence of Anemia and Its Associated Risk Factors Among 6-Months-Old Infants in Beijing. Frontiers in Pediatrics, 7, 286. https://doi.org/10.3389/fped.2019.00286

29. Urrechaga, E., Borque, L., \& Escanero, J. F. (2013). Biomarkers of hypochromia: the contemporary assessment of iron status and erythropoiesis. BioMed Research International, 2013, 603786. https://doi.org/10.1155/2013/603786

30. Deuel J., Lutz H., Misselwitz B., Goede J. Asymptomatic elevation of the hyperchromic red blood cell subpopulation is associated with decreased red cell deformability. Annals of Hematology. 2012; 91: 1427-1434. https://doi.org/10.1007/s00277012-1467-5

31. Maes M., Bosmans E., Scharpe S., Hendriks D., Cooremans W., Neels H., Meyer F.D., d'Hondt P., Peeters D. Components of biological variation in serum soluble transferrin receptor: relationships to serum iron, transferrin and ferritin concentrations, and immune and haematological variables. Scandinavian Journal of Clinical and Laboratory Investigation. 1997; 57(1): 31-41.

32. Keohane E.M., Otto C.N., Walenga J.M. Rodak's Hematology-E-Book: Clinical Principles and Applications. Elsevier Health Sciences. 2019: 550 p.

33. Christopher M.M., Hawkins M.G., Burton A.G. Poikilocytosis in rabbits: prevalence, type, and association with disease. PloS One. 2014; 9(11).

34. Chervier C., Cadore J.L., Rodriguez-Pineiro M.I., Deputte B.L., Chabanne L. Causes of anaemia other than acute blood loss and their clinical significance in dogs. The Journal of Small Animal Practice. 2012; 53(4): 223-227. https://doi.org/10.1111/j.17485827.2011 .01191

35. Chikazawa S., Dunning M.D. A review of anaemia of inflammatory disease in dogs and cats. The Journal of Small Animal Practice. 2016; 57(7): 348-353. https://doi.org/10.1111/jsap.12498 
36. Danchuk O.V. Lipid peroxidation and activity of the antioxidant protection system in pigs with different types of higher nervous activity. - The dissertation on competition of a scientific degree of the doctor of veterinary sciences on a specialty 03.00.13. "Human and animal physiology". - Kyiv, 2018. - 423 p. [In Ukrainian].

37. Stanley E., Appleman E., Schlag A., Siegel A. Relationship between cobalamin and folate deficiencies and anemia in dogs. Journal of Veterinary Internal Medicine. 2019; 33(1): 106-113.

38. Baldwin K., Bartges J., Buffington T., Freeman L.M., Grabow M., Legred J., Ostwald Jr. D. AAHA nutritional assessment guidelines for dogs and cats. Journal of the American Animal Hospital Association. 2010; 46(4): 285-296.

39. Aiken C.E.M., Allen N.B., Aryal S., Attarian H., Avula U.M.R., Bolignano D., Bradbury N.A., Burns J., Cahill L., Carcea I., Chan Y.Y., Chang H., Charkoudian N., Christiansen D.M., Coffey S.S., Coleman E., Cuttino M., Dobs A., Dorak M.T., Yang J.H. In M. J. Legato (Ed.), Principles of Gender-Specific Medicine (Third Edition) Academic Press. 2017; 600 p. https://doi.org/https://doi.org/10.1016/B978-0-12-803506-1.00060-7

40. Zullo S., Caenazzo L. Gene editing and gender-specific medicine: a challenge for dementia research. Palgrave Communications. 2020; 6(1): 1-6. 